\title{
Correlation of Pelvic Parameters with Isthmic Spondylolisthesis
}

\author{
Sung-Kyun Oh*, Sung-Soo Chung ${ }^{\dagger}$, Chong-Suh Lee ${ }^{\dagger}$ \\ *Department of Orthopedic Surgery,Wonkwang University Sanbon Hospital, Gunpo, Korea \\ ${ }^{\dagger}$ Department of Orthopedic Surgery, Samsung Medical Center, \\ Sungkyunkwan University School of Medicine, Seoul, Korea
}

Study Design: A retrospective radiological evaluation.

Purpose: To verify that PI is related with progression of IS as well as development of IS and to assess the differences of pelvic parameters between the L4 \& L5 IS, as well as between single $\&$ two level IS.

Overview of Literature: High pelvic incidence (PI) has been known to be related with development of IS. However, the previous studies were limited to just L5 spondylolisthesis or there was no differentiation between L4 \& L5 spondylolisthesis

Methods: Sixty five IS patients and 30 persons as a control group participated the study. Among the 65 patients, 30 had L4 IS, 30 had L5 IS and 5 had bi-level IS. We used the whole spine lateral radiographs to measure the slip percentage, the pelvic tilt (PT) and the pelvic incidence (PI), and we compared them between the normal control group and the IS patients, as well as between single-level and bi-level spondylolisthesis, and we investigated the correlation between the degree of slip of spondylolisthesis and the pelvic parameters.

Results: The averages of the PT, PI and lumbar lordosis (LL) in the control group and the IS group were $11.0^{\circ}$ vs $21.4^{\circ}$ (p<0.001), 49.1 vs $61.8^{\circ}$ (p<0.001) and $48.5^{\circ}$ vs $57.6^{\circ}$ (p<0.001), respectively. On comparison between the L4 and L5 IS groups, there was no difference in all the pelvic parameters (p>0.05). On comparison between the single-level IS group and the bilevel IS group, there was a significant difference of the PT and PI (p<0.05), and the slip percentage had a correlation with only the PI among all the pelvic parameters (Spearman's $r=0.293, p=0.023$ ). There was a significant correlation of the degree of slip with the PI for the L5 single level IS, but not with the L4 single level IS ( $r=0.362, p=0.05$ ).

Conclusions: The high pelvic incidence can be a factor of L4 \& L5 spondylolysis and it may have an influence on the slip progression in patients with L5 isthmic spondylolisthesis, but not on the slip progression in patients with L4 IS. Yet other factors seem to have an influence on the slip progression in patients with L4 isthmic spondylolisthesis.

Key Words: Isthmic spondylolisthesis, Pelvic parameter, Sacral slope, Pelvic tilt, Pelvic incidence

\section{Introduction}

The pelvic incidence (PI) angle was originally described by Beaupère et al. and it has been recently studied by many investigators ${ }^{1-3}$. The focus has primarily been on the sagittal alignment of the spine and pelvis ${ }^{3-6}$, Normal subjects, patients with scoliosis and those patients with isthmic spondylolisthesis were analyzed in their study $\mathrm{y}^{4,-9}$. The nor- mal patients were divided into the pediatric, adolescent, adult and elderly groups ${ }^{46}$. Duval-Beaupere et al. ${ }^{1}$ suggested that the PI has a positive correlation with lumbar lordosis and it is closely related with the sacral slope and pelvic tilt.

In the several studies that compared the PI between normal subjects and the patients with isthmic spondylolisthesis, it was reported that the PI was increasing as the grade of spondylolisthesis increased $^{7-9}$. Since an increased PI could be one of the causative factors in the development and pro-

Received Mar 4, 2009; 1st revised Apr 1, 2009; 2nd revised Apr 24, 2009; accepted Apr 26, 2009

Corresponding author: Chong-Suh Lee, MD

Department of Orthopedic Surgery, Samsung Medical Center, Sungkyunkwan University School of Medicine

50 Ilwon-dong, Kangnam-gu, Seoul, 135-710, Korea

Tel: +82-2-3410-3503 Fax: +82-2-3410-0061, E-mail: cs1@smc.samsung.co.kr 
gression of spondylolisthesis, PI could be one of the parameters to check when evaluating and treating spondylolisthesis. However, the previous studies were limited to just L5 spondylolisthesis or there was no differentiation between L4 \& L5 spondylolisthesis.

The purposes of this study is to assess the differences of the pelvic parameters between a normal control group and an isthmic spondylolisthesis group and between the groups of patients with L4 and L5 spondylolisthesis, as well as between groups of patients with single and bi-level spondylolisthesis. We then compared the groups to understand the correlation between the degree of slip of spondylolisthesis and the pelvic parameters.

\section{Materials and Methods}

\section{Materials}

A cohort of 65 patients who were operated on for treating their spondylolisthesis from March 2000 to August 2004 and 30 normal adults were recruited for this study. Of these spondylolisthesis patients, 30 subjects had spondylolisthesis at L4, 30 subjects had spondylolisthesis at L5 and 5 subjects had bi-level spondylolisthesis. Of these bi-level spondylolisthesis patients, 3 subjects had spondylolisthesis at both L3 and L4, and another 2 subjects had spondylolisthesis at both L4 and L5. The average age of the spondylolisthesis patients was 56.2 years (range, 24 76 years). Seventeen subjects were male and their average age was 59.9 years, while 48 subjects were female and their average age was 54.8 years. The normal control group consisted of 17 males and 13 females with an average age of 34.3 years (range, 28 42 years) and they were normal volunteers who worked in our hospital, and they had no history of severe back pain or spinal trauma.

\section{Radiologic examination and the method used to measure the pelvic parameters}

For each subject, the whole spine standing lateral films that included both hip joints were obtained with the subject in a standing position and their arms folded on their chest. To obtain a whole spine AP, the subject was in a standing position while placing their knees side by side. We recorded the degree of spondylolisthesis as a percentile according to the Taillard method, the degree of lumbar lordosis was assessed by Cobb's method from the upper endplate of L1 to upper endplate of S1, and the sacral slope (SS), pelvic tilt (PT) and PI were assessed by the method of Duval-Beaupere et al. ${ }^{1}$. According to the method of Duval-Beaupere et al. ${ }^{1}$, the SS was defined as the angle between the horizontal line and the sacral upper endplate, the PT was defined as the angle between the vertical line and the line joining the midpoint of the sacral upper endplate and the axis of the femoral heads, the PI was defined as the angle between a line joining the center of the upper endplate of $\mathrm{S} 1$ to the axis of the femoral heads and a line perpendicular to the upper endplate of S1 (Fig. 1). According to the geometrical analysis, the PI is the summation of the SS and the PT (Fig. 2).

\section{Statistical method}

The comparison of the average pelvic incidence between
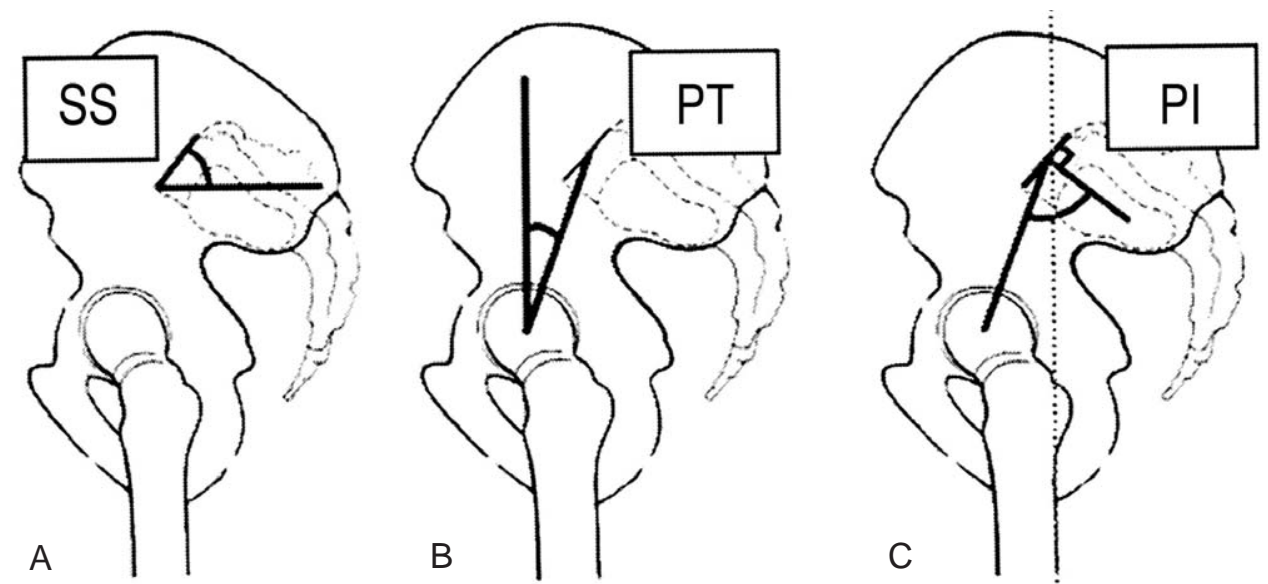

Fig. 1. Duval-Beaupère's pelvic parameters (A) sacral slope angle (SS), (B) pelvic tilt (PT) and (C) pelvic incidence (PI). 


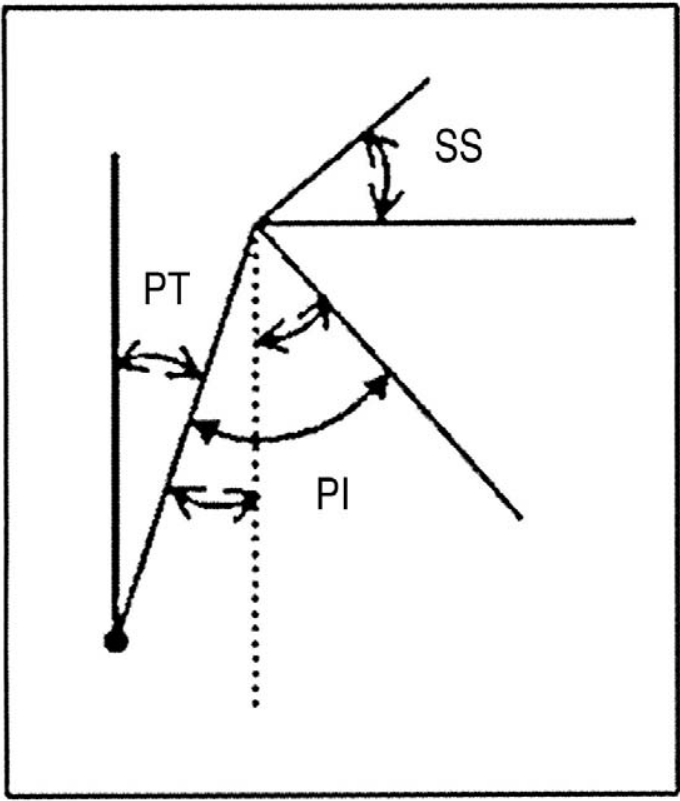

Fig. 2. Mathematical relation between the pelvic parameters: $\mathrm{PI}=\mathrm{PT}+\mathrm{SS}$.

the patients with isthmic spondylolisthesis, the normal controls and the patients with L4 and L5 spondylolisthesis were analyzed by student's t-test, while comparison of the average pelvic incidence between the patients with single-level spondylolisthesis and those with bi- level spondylolisthesis were analyzed by the Mann-Whitney U test. The correlation between the slip percentage and the pelvic incidence was analyzed by Spearman's correlation analysis (SPSS for windows version 11.5; SPSS Inc., Chicago, IL, USA). The statistical significance level was set at $\mathrm{p}$ values $<0.05$.

\section{Results}

\section{Comparison of the pelvic parameters between the isthmic spondylolisthesis and control groups}

In the control group, the SS was $38.1 \pm 7.3^{\circ}$, the PT was $11.0 \pm 5.9^{\circ}$, the PI was $49.1 \pm 8.8^{\circ}$ and the LL was $48.5 \pm$ $10.9^{\circ}$, and in the patient group the SS was $40.4 \pm 8.3^{\circ}$, the PT was $21.4 \pm 9.2^{\circ}$, the PI was $61.8 \pm 10.9^{\circ}$ and the lumbar lordosis (LL) was $57.6 \pm 11.4^{\circ}$. Of these parameters, the PT $(\mathrm{p}<0.001)$, PI $(\mathrm{p}<0.001)$ and LL $(\mathrm{p}<0.001)$ showed significant statistical differences (Table 1). Of the L4 spondylolisthesis patients, the averages of the SS, PT, PI and LL were $39.6 \pm 5.3^{\circ}, 20.5 \pm 7.7^{\circ}, 60.1 \pm 8.9^{\circ}$ and $59 \pm 8.4^{\circ}$, respectively, and for the L5 spondylolisthesis patients, the averages of the SS, PT, PI and LL were $41.8 \pm 10.3^{\circ}, 20.2 \pm$ $8.6^{\circ}, 62.0 \pm 12.5^{\circ}$ and $57 \pm 13.1^{\circ}$, respectively. The average slip percentages were $25.4 \pm 10.4 \%$ and $23.1 \pm 14.3 \%$ in the L4 and L5 spondylolisthesis patients, respectively; however, there was no statistical difference between the groups. ( $>0.05$, Table 2). For the single-level spondylolisthesis

Table 1. Comparison between the isthmic spondylolisthesis (IS) group and the control group for the pelvic parameters

\begin{tabular}{lccccc}
\hline & No. & SS & PT & PI & LL \\
\hline IS group & 65 & $40.3 \pm 8.3$ & $21.5 \pm 9.2$ & $61.7 \pm 10.9$ & $57.6 \pm 11.4$ \\
Control group & 30 & $38.1 \pm 7.3$ & $11.0 \pm 5.9$ & $49.1 \pm 8.8$ & $48.5 \pm 10.9$ \\
p-value & & 0.208 & $<0.001$ & $<0.001$ & $<0.001$ \\
\hline
\end{tabular}

SS: sacral slope, PT: pelvic tilt, PI: pelvic incidence, LL: lumbar lordosis.

Table 2. Comparison between the L4 IS group and the L5 IS group for the pelvic parameters

\begin{tabular}{lcccccc}
\hline & No. & SS & PT & PI & LL & Slip degree \\
\hline L4 IS & 30 & $39.6 \pm 5.3$ & $20.5 \pm 7.7$ & $60.1 \pm 8.9$ & $59.0 \pm 8.4$ & $25.4 \pm 10.4$ \\
L5 IS & 30 & $41.8 \pm 10.3$ & $20.2 \pm 8.6$ & $62.0 \pm 12.5$ & $57.0 \pm 13.1$ & $23.1 \pm 14.4$ \\
p-value & & 0.309 & 0.900 & 0.500 & 0.481 & 0.484 \\
\hline
\end{tabular}

IS: isthmic spondylolisthesis, SS: sacral slope, PT: pelvic tilt, PI: pelvic incidence, LL: lumbar lordosis.

Table 3. Comparison between the one-level IS group and the two-level IS group for the pelvic parameters

\begin{tabular}{lcccccc}
\hline & No. & SS & PT & PI & LL & Slip degree \\
\hline one-level IS & 60 & $40.7 \pm 8.2$ & $20.3 \pm 8.1$ & $61.0 \pm 10.8$ & $58.0 \pm 10.9$ & $24.3 \pm 12.5$ \\
two-level IS & 5 & $36.2 \pm 9.4$ & $34.8 \pm 11.8$ & $71.0 \pm 7.7$ & $52.8 \pm 16.8$ & $20.2 \pm 9.4$ \\
p-value & & 0.330 & 0.009 & 0.037 & 0.367 & 0.641 \\
\hline
\end{tabular}

IS: isthmic spondylolisthesis, SS: sacral slope, PT: pelvic tilt, PI: pelvic incidence, LL: lumbar lordosis. 
group, the averages of the SS, PT, PI and LL were $40.7 \pm$ $8.2^{\circ}, 20.3 \pm 8.1^{\circ}, 61.0 \pm 10.8^{\circ}$ and $58.0 \pm 10.9^{\circ}$, respectively, and for the bi-level spondylolisthesis group, the averages of the SS, PT, PI and LL were $36.2 \pm 9.4^{\circ}, 34.8 \pm 11.8^{\circ}$, $71.0 \pm 7.7^{\circ}$ and $52.8 \pm 16.8^{\circ}$, respectively. Of these parameters, the PT $(\mathrm{p}=0.009)$ and PI $(\mathrm{p}=0.037)$ showed significant differences (Table 3 ).

\section{Analysis of the correlation between the slip per- centage and the pelvic parameters in the patients with single level spondylolisthesis}

We analyzed the correlation between the slip percentage and the other pelvic parameters (SS, PI, PT, LL). For the 60 single-level spondylolisthesis group, the correlation coefficient between the slip percentage and the SS was $r=0.121$ $(\mathrm{p}=0.356)$, the correlation coefficient between the slip percentage and the PT was $\mathrm{r}=0.222(\mathrm{p}=0.088)$, the correlation coefficient between the slip percentage and the PI was $\mathrm{r}=0.293(\mathrm{p}=0.023)$ and that between the slip percentage and the LL was $r=0.132(p=0.315)$. Only the PI showed statistically significant correlation (Table 4). For the L4 spondylolisthesis group, the correlation coefficient between the slip percentage and the SS was $0.116(\mathrm{p}=0.543)$, that between the slip percentage and the PT was $r=0.268(\mathrm{p}=0.152)$, that between the slip percentage and the PI was $r=0.153$ $(\mathrm{p}=0.421)$ and that between the slip percentage and the LL was $r=0.05(p=0.795)$ There were no pelvic parameters that showed significant correlation with the slip percentage in the L4 spondylolisthesis group (Table 4). For the entire L5 spondylolisthesis group, the correlation between the slip percentage and the SS was $\mathrm{r}=0.305(\mathrm{p}=0.101)$, that between the slip percentage and the PT was $r=0.183(p=0.334)$, that between the slip percentage and the PI was $r=0.362$ $(\mathrm{p}=0.050)$ and that between the slip percentage and the LL was $r=0.144(p=0.447)$. Only the PI showed significant statistical correlation with the slip percentage (Table 4).

\section{Discussion}

The causes of spondylolysis are considered to be multifactorial, and they include a hereditary predisposition and biomechanical factors. The precise mechanism of slippage after the development of an isthmic defect is unknown, yet there are many theoretical explanations for slip progression. The slip angle, slip grade and sacral inclination had been reported to be important parameters by which we can predict the slip progression. The sagittal pelvic tilt index was first suggested by Schwab et al. ${ }^{10}$ to be an index that explains the positional relations between L5 and the hip axis, and it specifies the degree of the anteroposterior rotation of the pelvis. Schwab et al. ${ }^{10}$ described rotation of the pelvis as developing with verticalization of the sacrum and anterior displacement of the hip joint, and this suggests a progression of spondylolisthesis by this index. A verticalization of the sacrum and anterior displacement of the hip joint is the same phenomenon as an increasing PT, which can be seen in this study. This develops as a compensatory mechanism for the anterior displacement of the vertebral body from the gravity line by the spondylolisthesis, but it is difficult to consider it as a parameter to predict the progression of spondylolisthesis.

The pelvic incidence was suggested by Duval-Beaupere et al. ${ }^{1}$ to be an anatomical parameter that is correlated with such positional parameters as the sacral slope and pelvic tilt. Therefore, as the PI is increased, the PT and SS will be increased. Generally, the normal range of the PT is very narrow from 10 to 15 degrees, so if the PI is increased, the SS will be increased relatively much more than the PT and it will be the cause of increasing lordosis. If the lordosis is increased, there will be a large load to the posterior complex that will produce a spondylolysis and possible progression of spondylolisthesis. Progression of spondylolisthesis

Table 4. Correlation coefficient between the degree of slip and the other pelvic parameters in one-level IS, L4 IS and L5 IS

\begin{tabular}{lcccccc}
\hline & No. & Paremeter & SS & PT & & PI \\
\hline One-level IS & 60 & Slip degree & 0.121 & 0.222 & $0.293 *$ & 0.132 \\
& & p-value & 0.356 & 0.088 & 0.023 & 0.315 \\
L4 IS & \multirow{2}{*}{30} & Slip degree & -0.116 & 0.268 & 0.153 & 0.05 \\
& & p-value & 0.543 & 0.152 & 0.421 & 0.795 \\
L5 IS & \multirow{2}{*}{30} & Slip degree & 0.305 & 0.183 & $0.362 *$ & 0.144 \\
& & p-value & 0.101 & 0.334 & 0.050 & 0.447 \\
\hline
\end{tabular}

*Correlation is significant at the 0.05 level (2-tailed). 
displaces the center of gravity of the pelvis anteriorly, which increases the PT and decreases the SS to compensate.

On comparison of the pelvic parameters between 48 subjects with isthmic spondylolisthesis and 30 normal subjects by Rajinics et al. ${ }^{9}$, the SS, PT and PI in the spondylolisthesis group were significantly higher than those values in the normal group. In their study, the results for the PI were 66.5 degrees in the spondylolisthesis group and 54.0 degree in the normal group, and they described a correlation between the slip percentage and the PI ( $\mathrm{r}=0.660)$. In their study, although $15 \mathrm{~L} 4$ spondylolisthesis subjects were included, the analysis was done without differentiating between L4 and L5 spondylolisthesis. However in our study, there was no statistical difference of the SS between the spondylolisthesis group \& the normal control group, but a difference of the PI was present, which applied to the difference of the PT.

Hanson et al. ${ }^{7}$ measured the pelvic parameters in 40 patients with isthmic spondylolisthesis and 40 normal subjects. There were 20 children and 20 adults in the group of normal subjects with an average age of 11.8 years and 60.0 years, respectively. They classified the Meyerding-Newman grades 1 and 2 into the mild spondylolisthesis group with grades 3 and 4 into the high grade spondylolisthesis group, and the average age of the groups was 26.6 years and 17.7 years, respectively. In their study, the average PI was $47.4^{\circ}$ in the children's group, $57.0^{\circ}$ in the adult's group, $68.5^{\circ}$ in the mild spondylolisthesis group and $79.0^{\circ}$ in the high grade spondylolisthesis group. The PI of the high grade spondylolisthesis group was greatest, and the PI of the mild spondylolisthesis group was second greatest. Additionally, there was a correlation between the grade of spondylolisthesis and the PI by the Meyerding-Newman score $(\mathrm{p}=0.03)$. However, in their study, there was no analysis of the SS and PT as a function of the PI, so there was no data on the differences of the SS and PT between the two groups.

Labelle et al. ${ }^{8}$ measured the pelvic parameters in 214 patients with L5 isthmic spondylolisthesis, along with a control group of 160 normal subjests. These patients were classified into 5 groups by the Meyerding classification; on comparing each group, the PI, SS, PT and LL were found to be higher in the isthmic spondylolisthesis group. Additionally, as the degree of spondylolisthesis increased, the LL, PI and PT increased as well.

In our study, the PI in the spondylolisthesis group was higher than that in the normal group $\left(61.8^{\circ}\right.$ and $49.1^{\circ}$, respectively, $\mathrm{p}<0.01$ ), and the PT in the spondylolisthesis group was higher than in the normal group $\left(21.4^{\circ}\right.$ and $11.0^{\circ}$, respectively, $\left.\mathrm{p}<0.01\right)$. These results were in accord with the other reported results. Labell et al.'s conclusion that the SS of the spondylolisthesis group was higher than that of the normal group was not supported by our results. We estimated that the reason why the PT was increased, but the SS was not increased in our study was due to the compensatory increased pelvic tilt in the patients with spondylolisthesis. So it ultimately reduced the SS in the spondylolisthesis group and it made no difference between the study and the control group with the difference of the PI primarily depending on the PT. This can be more clearly seen in bi-level spondylolistheis, which has a greater PI and PT than one-level spondylolisthesis without any difference in the SS between the two groups. Our study had the age factor as a significant difference from Labelle's study, where the average age in his study was 16.8 years and the average age in our study was 56.2 years. It is generally assumed that the PT increases and the SS decrease with increasing age, which can be another reason for the lack of differences of the SS between the spondylolisheis group and the normal control group in our study.

The studies by Rajinics, Hanson, and Labelle have reported a correlation between the slip percentage and the PI according to the Meyerding grade ${ }^{7-9}$. In our study, we analyzed the correlations of the pelvic parameters with the slip percentage in 60 single level spondylolisthesis subjects by the Taillard method, with the result that there was a correlation between the slip percentage and the PI $(r=0.293$, $\mathrm{p}=0.023$ ), and there was no correlation among the other parameters $(p>0.05)$. We believe this fact can provide a theoretical basis for concluding that a high PI is correlated with slip progression. There was, however, only correlation between the pelvic incidence and the displacement in the L5 spondylolisthesis group $(\mathrm{r}=0.362, \mathrm{p}=0.05)$, but not in the $\mathrm{L} 4$ spondylolisthesis group. In our studies, we could assume the reason we couldn't see correlation between the slip percentage and the pelvic parameters in L4 IS is that the anatomical configuration of the pelvis has an influence on the progression of slippage in L5, but it didn' $t$ influence on the slippage of L4. L5 is more stable than L4 due to the iliolumbar or iliotransverse ligament and the protected position of L5 below the iliac crest. In L4 spondylolisthesis, there might be other different independent factors such as segmental instability or concurrent disc degeneration at the level of the slip, which can have a greater influence on slip progression. Also, our results showed that there were no 
differences in the pelvic parameters between L4 and L5 spondylolisthesis.

However, the other result that the PI and PT were larger in two level spondylolisthesis than in one-level spondylolisthesis shows that pelvic parameters may have an influence on the slip progression of L4 IS, but we couldn't totally conclude that L4 IS is different from L5 IS. Therefore, although a high PI can be associated with the occurrence of spondylolysis at L4 and L5, L5 is regarded as more related to the progression of slip with L5's higher PI than that of L4.

Ultimately, even though there were limitations of our study, which include some young patients, we believe this subject needs further studies, including studies that will make comparisons not with a normal group, but with nonprogressed sponlylolysis patients.

\section{Conclusions}

A high pelvic incidence can be a factor for spondylolysis and it can have an influence on slip progression in patients with L5 isthmic spondylolisthesis. However, other factors seem to have an influence on slip progression in patients with L4 isthmic spondylolisthesis.

\section{REFERENCES}

1. Duval-Beaupere G, Schmidt C, Cosson P: A Barycentremetric study of the sagittal shape of spine and pelvis: the conditions required for an economic standing position. Ann Biomed Eng 1992; 20: 451-462.
2. Curylo LJ, Edwards C, DeWald RW: Radiographic markers in spondyloptosis: implications for spondylolisthesis progression. Spine (Phila Pa 1976) 2002; 27: 20212025 .

3. Hammerberg EM, Wood KB: Sagittal profile of the elderly. J Spinal Disord Tech 2003; 16: 44-50.

4. Legaye J, Duval-Beaupere G, Hecquet J, Marty C: Pelvic incidence: a fundamental pelvic parameter for threedimensional regulation of spinal sagittal curves. Eur Spine J 1998; 7: 99-103.

5. Mac-Thiong JM, Berthonnaud E, Dimar JR 2nd, Betz RR, Labelle H: Sagittal alignment of the spine and pelvis during growth. Spine (Phila Pa 1976) 2004; 29: 1642-1647.

6. Vaz G, Roussouly P, Berthonnaud E, Dimnet J: Sagittal morphology and equilibrium of pelvis and spine. Eur Spine J 2002; 11: 80-87.

7. Hanson DS, Bridwell KH, Rhee JM, Lenke LG: Correlation of pelvic incidence with low- and high-grade isthmic spondylolisthesis. Spine (Phila Pa 1976) 2002; 27: 20262029.

8. Labelle H, Roussouly P, Berthonnaud E, et al: Spondylolisthesis, pelvic incidence, and spinopelvic balance: a correlation study. Spine (Phila Pa 1976) 2004; 29: 20492054.

9. Rajnics P, Templier A, Skalli W, Lavaste F, Illes T: The association of sagittal spinal and pelvic parameters in asymptomatic persons and patients with isthmic spondylolisthesis. J Spinal Disord Tech 2002; 15: 24-30.

10. Schwab FJ, Farcy JP, Roye DP Jr: The sagittal pelvic tilt index as a criterion in the evaluation of spondylolisthesis. Preliminary observations. Spine (Phila Pa 1976) 1997; 22: 1661-1667. 